\title{
Immune checkpoint inhibitors and small cell lung cancer: what's new?
}

\author{
Sabine Schmid, Martin Früh \\ Department of Oncology, Haematology, Cantonal Hospital St. Gallen, St. Gallen, Switzerland \\ Contributions: (I) Conception and design: All authors; (II) Administrative support: None; (III) Provision of study materials or patients: None; (IV) \\ Collection and assembly of data: S Schmid; (V) Data analysis and interpretation: None; (VI) Manuscript writing: All authors; (VII) Final approval of \\ manuscript: All authors. \\ Correspondence to: Martin Früh. Department of Oncology and Haematology, Cantonal Hospital St. Gallen, Rorschacherstrasse 95, 9007 St. Gallen, \\ Switzerland. Email: martin.frueh@kssg.ch.
}

\begin{abstract}
Despite extensive research no meaningful progress in systemic treatment of small cell lung cancer (SCLC) has been made in the past decades. Earlier attempts with immunotherapy including interferon and vaccination approaches had limited success. High mutational load, smoking history and potentially also the frequent presence of paraneoplastic phenomena-indicating an activated immune system-represent a rationale for a benefit from immune checkpoint inhibitors in SCLC. However, the likelihood of response is diminished due to poor T-cell activation resulting from low expression of MHC class I antigens, low amounts of tumor infiltrating lymphocytes (TILs) and low PD-L1 expression rates. Recently, early reports from studies with checkpoint inhibitors have shown promising results with the potential for long term disease control in a subset of SCLC patients. However, reliable predictive biomarkers to better define the population drawing most benefit are currently lacking. Results from ongoing phase III trials in different treatment lines and in the maintenance setting are eagerly awaited.
\end{abstract}

Keywords: Small cell lung cancer (SCLC); checkpoint inhibitors; predictive biomarkers

Submitted Dec 22, 2017. Accepted for publication Jan 14, 2018.

doi: $10.21037 /$ jtd.2018.01.113

View this article at: http://dx.doi.org/10.21037/jtd.2018.01.113

\section{Introduction}

Lung cancer is the leading cause of cancer related mortality and an estimated 1.8 million new lung cancers are diagnosed worldwide each year. The proportion of small cell lung cancer (SCLC) is estimated around 13\%, with a history of tobacco use in literally all patients (1). Approximately $70 \%$ of the SCLC patients present with stage IV disease, where the treatment approach is palliative. Standard treatment in Western Countries in these patients is combination chemotherapy with either cisplatin or carboplatin and etoposide $(2,3)$. Although this regimen achieves high response rates (RR) of $65-70 \%$, outcomes are poor with a median overall survival (OS) of $<10$ months due to early drug resistance and rapid tumour progression (4). Despite extensive research no meaningful progress has been made with systemic treatment in the past decades. Repeated efforts of integrating immunotherapy into SCLC treatment have been made with only little success in the past years. These approaches included interferons, which enhance major histocompatibility complex (MHC) class I expression, but neither for the combination of low-dose interferon with chemotherapy in the first-line setting (5) nor for maintenance treatment with interferon alone or in combination with retinoid acid after induction chemotherapy or chemoradiotherapy have eventually demonstrated any significant benefit $(6,7)$. The same is true for vaccinations: ganglioside antigen (GD3) is overexpressed in SCLC in approx. $60 \%$ of cases (8). Therefore, Bec2 antiidiotypic antibody that mimics GD3 seemed a promising target, but a large phase III trial with the antiganglioside vaccine Bec2/Bacille Calmette-Guerin (Bec2/BCG) 
vaccination in the adjuvant setting after Chemotherapy and chest radiotherapy in limited stage SCLC did not show improved outcomes (9).

Early results from studies with immunotherapy checkpoint inhibitors now finally show some promising outcomes in a subset of patients with the potential for improvement of survival in a field where it is much needed $(8,10-12)$. We will focus our review on immune checkpoint inhibitors in patients with SCLC.

\section{Perspectives of immunotherapy in SCLC}

Mutational burden has been proposed as predictive biomarker for checkpoint inhibitor treatment in several tumour types including NSCLC (13). In line with previous studies comprehensive genomic profiling of 110 SCLC specimens showed very high mutation rates (14-16). Tumor-suppressor-genes TP53 and RB1 were altered in all but two cases supporting hypothesis that complete genomic loss of both TP53 and RB1 function is obligatory in the pathogenesis of SCLC, leading to increased genomic instability. In addition the carcinogenic effect of tobacco is associated with a higher mutational burden (10). In other tumour entities such as NSCLC, smoking-related lung cancers are characterized by a greater mutation burden compared to never smokers (17) and response to immunotherapy has consistently shown to be better in patients with a smoking history compared to never smokers in large phase III trials (18-20). Overall high mutational burden and the almost universal smoking history of SCLC patients would favour improved outcomes of immunotherapy in these patients.

Autoimmunological paraneoplastic syndromes including neurological and endocrinological phenomena are quite common in SCLC patients, suggesting an activated immune system in these patients. In a series of 170 SCLC patients presence of anti-Hu antibodies was an independent factor for complete response to therapy in a multivariate analysis and as opposed to the majority of SCLC patients, MHC class I proteins were not down-regulated if anti$\mathrm{Hu}$ antibodies were present, potentially rendering these patients more sensitive to immune response $(12,21,22)$. On the other hand, potential enhancement of response may also imply higher risk for neurological autoimmune phenomena as encephalitis and myasthenia gravis (23). Therefore, toxicity needs to be closely monitored, as in other tumour entities that are typically accompanied with paraneoplastic phenomena such as thymomas where serious autoimmune related side effects have been observed (24).

Tumour-infiltrating lymphocytes (TILs) in the tumour as potential predictive biomarker for checkpoint inhibition are less common in SCLC and are mostly found at the tumour periphery at the border of tumour and stroma and not within the tumour $(25,26)$.

One reason for the relatively marginal $\mathrm{T}$-cell recruitment in SCLC could be the reduced or absent MCH class I expression on SCLC tumor cells. Tumor antigens are presented to the immune system by MHC class I surface antigens. Reduced or absent MHC class I expression leads to decreased antigen presentation to cytotoxic T-cells (CTLs) (25). In SCLC cell lines and fresh tumor samples reduced expression of MHC class I surface antigens could be shown despite intact MHC class I genes, indicating a block in mRNA transcription (27), unlike it is the case in NSCLC cell lines. Interferons are known to augment MHC class I in melanoma cells (28) and Doyle et al. could show, that interferons also can increase HLA expression in SCLC cell lines, where these have been markedly decreased (27). Balance of $\mathrm{T}$ effector cells (Teff) and T regulatory cells (Treg) seems to play an important role in SCLC as Teff/ Treg-ratio corresponds with risk of recurrence in an analysis of 35 patients with SCLC with long-term survivors maintaining a high ratio of Teff to Treg cells and patients with recurrent disease a low ratio, respectively (29). Also, high number of lymphocytes in the peripheral blood correlated with better survival (30). In summary low expression of $\mathrm{MCH}$ class I antigens leads to decreased T-cell recruitment, explaining low numbers of TILs within the tumor in SCLC patients, being a negative predictive marker for response to immunotherapy.

Programmed death-ligand 1 (PD-L1) expression status of tumor cells is used to identify patients who might be more likely to benefit from immune checkpoint inhibitors. However, PD-L1 status is influenced by various factors such as intratumoral heterogeneity, dynamic changes over time and the availability of various different test methods using different thresholds for PD-L1 positivity $(31,32)$. In SCLC, data on PD-L1 expression are controversial but overall expression seems to be quite low. In the recent checkmate- 032 trial only $18 \%$ of patients had a PD-L1 expression $>1 \%$ and expression status was not relevant for response to treatment and outcomes (23). In an analysis of 94 small cell specimens (61 of the lung, rest extrapulmonal) analyzed for PD-L1 expression on tumor cells and microenvironment none of the analysed tumor cells stained positive for PD-L1, whereas in $18.5 \%$ of cases 
PD-L1 positive cells (mainly monocytes) in the adjacent stroma were found. In $48 \%$ of cases PD-1 positive TILs were found, although not also TILs were seen mainly at the border of tumor and stroma, not within the tumor (26). This is supported also by a study by Rivalland et al. presented at ASCO 2017, where in 105 SCLC specimens analysed tumor cells positive for PD-L1 expression were only found in a minority of cases (18\%) (33). On the other hand, other studies demonstrated high PD-L1 expression in SCLC specimens (34-36).

In conclusion, although high mutational load and presence of paraneoplastic phenomena represent a rationale for potential benefit of immunotherapy in SCLC patients, latter may harbour the risk of increased autoimmunological side effects. In addition, T-cell activation is poor due to low expression of MHC class I antigens and TILs are uncommon in the vast majority of SCLC specimens as is the relatively low rate of PD-L1 expression lowering the likelihood of response to immune checkpoint inhibitors.

\section{Clinical trials with immune checkpoint inhibitors in SCLC}

\section{First line treatment}

First promising signs of activity of checkpoint inhibitors as novel immunotherapeutic approach came from a phase II trial of the CTL4-antibody ipilimumab in combination with carboplatin and taxol first-line in extensive stage SCLC with improved progression-free survival (PFS) compared to chemotherapy alone when given in a "phased" fashion meaning the introduction of immunotherapy after 2 cycles of chemotherapy (37). However, the following phase III trial was negative regarding its primary endpoint overall survival (OS) for the combination of platinum/etoposide in combination with ipilimumab which was again administered as phased treatment in the same patient population (38). Nevertheless, a similar approach is now being investigated targeting the PD-1/PD-L1 axis amongst others in three large trials: KEYNOTE-604 comparing pembrolizumab plus etoposide/platinum to chemotherapy alone (NCT03066778), IMpower133 which compares atezolizumab with carboplatin and etoposide to chemotherapy alone (NCT02763579) and CASPIAN studying durvalumab alone or in combination with tremelimumab with platinum based chemotherapy followed by durvalumab \pm tremelimumab maintenance therapy versus chemotherapy alone (NCT03043872), all in the first-line setting.

\section{Maintenance treatment}

Initial high tumor burden and the often-rapid response with chemotherapy in the $1 \mathrm{~L}$-setting followed by early drug resistance and rapid tumour progression theoretically favour the investigation of maintenance treatment regimens to delay progression and improve overall survival. At ASCO 2017 a phase II study of maintenance pembrolizumab in 45 patients with extensive stage small cell lung cancer was presented, however no improvement in PFS could be demonstrated (39). Checkmate-451, a randomized placebo-controlled phase III trial investigating a similar approach with nivolumab alone or in combination with ipilimumab finished recruiting (NCT02538666). The phase II IMPULSE trial investigating maintenance treatment with the TLR9 agonist Lefitolimod has finished recruiting, results are awaited NCT02200081.

\section{Second or later line treatment}

For treatments targeting the PD-1/PD-L1 axis first reports for activity came from multi-cohort studies including SCLC cohorts: Keynote- 028 , a phase $1 \mathrm{~b}$ multi-cohort trial with pembrolizumab as well as Checkmate-032, a phase I/II trial with nivolumab alone or the combination of nivolumab and ipilimumab.

Results from the SCLC cohort of Keynote-028, which only included PD-L1 positive $(>1 \%)$ patients, were first reported at the WCLC 2016 by Ott et al. and recently published (40). Twenty-four SCLC patients with extensive disease and at least one prior treatment line were treated with pembrolizumab $10 \mathrm{mg} / \mathrm{kg}$ every 2 weeks. In this cohort confirmed overall response rate (ORR) was 33\% with one complete remission (CR) and median duration of response was 19.4 months. OS was promising in this heavily pre-treated patient population with 9.7 months with a 12 -month OS rate of almost $40 \%$. However, although promising survival data were observed in second line also including poor prognostic platinum resistant patients, this trial was very small and the subset of likely highly selected responders consisted of eight patients only, of whom five received treatment for over 1 year. Results of lager trials have to be awaited in order to draw definitive conclusions about the activity of single agent pembrolizumab in this situation. Trials with pembrolizumab with the current standard dose of $200 \mathrm{mg}$ every 3 weeks are ongoing in PDL1 unselected patients (KEYNOTE 158) as well as in the first-line and maintenance setting and in combination with 
chemotherapy or chemoradiotherapy (NCT03066778, NCT02359019, NCT02934503).

Another small phase Ia trial investigated the anti-PL-L1 monoclonal antibody atezolizumab as a monotherapy in 17 heavily pre-treated SCLC patients. The trial showed a confirmed ORR by RECIST in only 1 patient (6\%) (41).

Checkmate-032 was a larger phase I/II trial that enrolled a total of 216 patients who were unselected for PD-L1 status and progressing after at least one previous platinumcontaining regimen into three different SCLC cohorts (23). Patients were treated either with nivolumab $3 \mathrm{mg} / \mathrm{kg}$ every 2 weeks alone or in combination with ipilimumab in a safety dose-escalating manner with the dose of nivolumab $1 \mathrm{mg} / \mathrm{kg}$ and ipilimumab $3 \mathrm{mg} / \mathrm{kg}$ every 3 weeks followed by nivolumab $3 \mathrm{mg} / \mathrm{kg}$ every 2 weeks emerging as the regimen for succeeding studies due to favourable PFS of 2.6 months which was numerically higher than in the other cohorts (1.4 and 1.4 months). ORR was $25 \%$ in the combination-arm but only $11 \%$ in the nivolumab monotherapy arm with a median duration of response of 11.7 months. Median OS was markedly shorter than in the keynote 028 trial with 4.4 months for monotherapy and 7.9 months for the combination. However, the 2-year OS rate of $26 \%$ suggests that a subset of SCLC patients may derive long-term benefit. Outcomes in this trial were independent of PD-L1 expression, which was only positive in a minority of patients $(18 \%>1 \%$ PD-L1 expression) and unrelated to platinum-sensitivity. Interestingly, similarly to NSCLC (13) high tumor mutational burden (TMB) measured by whole exome sequencing emerged as possible predictive biomarker in an exploratory analysis presented at WCLC 2017 (42). TMB was classified into three groups based on number of nonsynonymous somatic mutations: high (>243 mutations), medium (100-243 mutations) and low ( $<100$ mutations). ORR to combination treatment was $46 \%$ in the TMB high group versus $16 \%$ and $22 \%$ in the intermediate and low groups. This was reflected also by a markedly higher 1-year PFS rate of $30 \%$ in the TMB high group receiving combination treatment versus 8 and $6 \%$ in the intermediate and low groups. The same tendencies were also shown for patients receiving monotherapy but ORR was lower than in the combination arms in all patients. Although these results are derived from a relatively small exploratory subgroup analysis $(\mathrm{n}=26)$, particularly 1 -year PFS rate in the TMB high group appear very promising and potentially identifies a proportion of patients who may have prolonged disease control in this poor prognostic group of patients. Overall, combination treatment seems more effective, but treatment toxicity has to been taken into account, particularly with the SCLC population outside of clinical trials who are often older and frail due to tobaccoassociated comorbidities. In the combination arm with nivolumab $1 \mathrm{mg} / \mathrm{kg}$ and ipilimumab $3 \mathrm{mg} / \mathrm{kg}(\mathrm{n}=61)$ the rate of adverse events (AE) was 79\% and grade 3-4 adverse events (AEs) occurred in as many as $30 \%$ of patients. Seven patients $(11 \%)$ discontinued treatment because of toxicity and two patients died due to treatment-related AEs. Importantly, neurological autoimmune events occurred in three patients having limbic encephalitis, including one patient with grade 4 not resolving despite i.v. corticosteroids and immunoglobulins, and one patient dying with myasthenia gravis, indicating that these side effects may be observed more frequently in these patients than in other malignancies.

A number of trials in the second-line setting are ongoing with a focus on combination therapies such as the phase $1 / 2$ trial investigating Rovalpituzumab Tesirine [a delta-like 3 (DLL3) directed anti-body drug conjugate] with nivolumab or in combination with both nivolumab and ipilimumab (NCT03026166) and the first in class Fucosyl-GM1 mAb, a fully human mAb with enhanced antibody-dependent cell-mediated cytotoxicity that binds to fucosyl-GM1, a ganglioside highly expressed on SCLC, in combination with nivolumab (NCT02247349).

\section{Limited stage}

Adjuvant treatment with ipilimumab and nivolumab after completion of curative chemoradiotherapy in limited stage SCLC is being evaluated in the ongoing ETOP STIMULI trial (NCT02046733), results are awaited 2020.

\section{Conclusions}

After decades without relevant progress in treatment of metastatic SCLC, early trial results indicate that immunotherapy with checkpoint inhibitors may have the potential for long-term disease control in a subset of patients. Results from currently ongoing large phase III trials in first and later lines as well as in the maintenance setting are eagerly awaited. Combination immunotherapy appears to be more active than single agent anti-PD-1/ PD-L1 therapy, although more toxic. Therefore, it will be particularly important to identify predictive markers in order to better select patients drawing benefit from treatment. Early results are indicating that high TMB 
may be of interest for selection of anti-PD-1/CTLA-4 combination. In this regard, further analyses of ongoing clinical trial are eagerly awaited.

\section{Acknowledgements}

None.

\section{Footnote}

Conflicts of Interest: The authors have no conflicts of interest to declare.

\section{References}

1. Govindan R, Page N, Morgensztern D, et al. Changing epidemiology of small-cell lung cancer in the United States over the last 30 years: analysis of the surveillance, epidemiologic, and end results database. J Clin Oncol 2006;24:4539-44.

2. Pujol JL, Carestia L, Daures JP. Is there a case for cisplatin in the treatment of small-cell lung cancer? A meta-analysis of randomized trials of a cisplatin-containing regimen versus a regimen without this alkylating agent. $\mathrm{Br} \mathrm{J}$ Cancer 2000;83:8-15.

3. Mascaux C, Paesmans M, Berghmans T, et al. A systematic review of the role of etoposide and cisplatin in the chemotherapy of small cell lung cancer with methodology assessment and meta-analysis. Lung Cancer 2000;30:23-36.

4. Foster NR, Qi Y, Shi Q, et al. Tumor response and progression-free survival as potential surrogate endpoints for overall survival in extensive stage small-cell lung cancer: findings on the basis of North Central Cancer Treatment Group trials. Cancer 2011;117:1262-71.

5. Ruotsalainen TM, Halme M, Tamminen K, et al. Concomitant chemotherapy and IFN-alpha for small cell lung cancer: a randomized multicenter phase III study. J Interferon Cytokine Res 1999;19:253-9.

6. Ruotsalainen T, Halme M, Isokangas OP, et al. Interferonalpha and 13-cis-retinoic acid as maintenance therapy after high-dose combination chemotherapy with growth factor support for small cell lung cancer--a feasibility study. Anticancer Drugs 2000;11:101-8.

7. Mattson K, Niiranen A, Ruotsalainen T, et al. Interferon maintenance therapy for small cell lung cancer: improvement in long-term survival. J Interferon Cytokine Res 1997;17:103-5.
8. Spigel DR, Socinski MA. Rationale for chemotherapy, immunotherapy, and checkpoint blockade in SCLC: beyond traditional treatment approaches. J Thorac Oncol 2013;8:587-98.

9. Giaccone G, Debruyne C, Felip E, et al. Phase III study of adjuvant vaccination with Bec2/bacille CalmetteGuerin in responding patients with limited-disease smallcell lung cancer (European Organisation for Research and Treatment of Cancer 08971-08971B; Silva Study). J Clin Oncol 2005;23:6854-64.

10. Bunn PA Jr, Minna JD, Augustyn A, et al. Small Cell Lung Cancer: Can Recent Advances in Biology and Molecular Biology Be Translated into Improved Outcomes? J Thorac Oncol 2016;11:453-74.

11. Chae YK, Pan A, Davis AA, et al. Recent Advances and Future Strategies for Immune-Checkpoint Inhibition in Small-Cell Lung Cancer. Clin Lung Cancer 2017;18:132-40.

12. Lehman JM, Gwin ME, Massion PP. Immunotherapy and Targeted Therapy for Small Cell Lung Cancer: There Is Hope. Curr Oncol Rep 2017;19:49.

13. Peters S, Creelan B, Hellmann MD, et al. Abstract CT082: Impact of tumor mutation burden on the efficacy of firstline nivolumab in stage iv or recurrent non-small cell lung cancer: An exploratory analysis of CheckMate 026. Cancer Res 2017;77:CT082.

14. Rudin CM, Durinck S, Stawiski EW, et al. Comprehensive genomic analysis identifies SOX2 as a frequently amplified gene in small-cell lung cancer. Nat Genet 2012;44:1111-6.

15. Peifer M, Fernandez-Cuesta L, Sos ML, et al. Integrative genome analyses identify key somatic driver mutations of small-cell lung cancer. Nat Genet 2012;44:1104-10.

16. George J, Lim JS, Jang SJ, et al. Comprehensive genomic profiles of small cell lung cancer. Nature 2015;524:47-53.

17. Govindan R, Ding L, Griffith M, et al. Genomic landscape of non-small cell lung cancer in smokers and neversmokers. Cell 2012;150:1121-34.

18. Hellmann M, Rizvi N, Wolchok JD, et al. Genomic profile, smoking, and response to anti-PD-1 therapy in non-small cell lung carcinoma. Mol Cell Oncol 2015;3:e1048929.

19. Borghaei H, Paz-Ares L, Horn L, et al. Nivolumab versus Docetaxel in Advanced Nonsquamous Non-Small-Cell Lung Cancer. N Engl J Med 2015;373:1627-39.

20. Rittmeyer A, Barlesi F, Waterkamp D, et al. Atezolizumab versus docetaxel in patients with previously treated non-small-cell lung cancer (OAK): a phase 3, openlabel, multicentre randomised controlled trial. Lancet 
2017;389:255-65.

21. Graus F, Dalmou J, Rene R, et al. Anti-Hu antibodies in patients with small-cell lung cancer: association with complete response to therapy and improved survival. J Clin Oncol 1997;15:2866-72.

22. Darnell RB, Posner JB. Paraneoplastic syndromes involving the nervous system. N Engl J Med 2003;349:1543-54.

23. Antonia SJ, Lopez-Martin JA, Bendell J, et al. Nivolumab alone and nivolumab plus ipilimumab in recurrent smallcell lung cancer (CheckMate 032): a multicentre, openlabel, phase 1/2 trial. Lancet Oncol 2016;17:883-95.

24. Giaccone G, Thompson JF, Crawford J, et al. A phase II study of pembrolizumab in patients with recurrent thymic carcinoma. J Clin Oncol 2017;35:8517.

25. Blank CU, Haanen JB, Ribas A, et al. CANCER IMMUNOLOGY. The "cancer immunogram". Science 2016;352:658-60.

26. Schultheis AM, Scheel AH, Ozretic L, et al. PD-L1 expression in small cell neuroendocrine carcinomas. Eur J Cancer 2015;51:421-6.

27. Doyle A, Martin WJ, Funa K, et al. Markedly decreased expression of class I histocompatibility antigens, protein, and mRNA in human small-cell lung cancer. J Exp Med 1985;161:1135-51.

28. Basham TY, Bourgeade MF, Creasey AA, et al. Interferon increases HLA synthesis in melanoma cells: interferonresistant and -sensitive cell lines. Proc Natl Acad Sci U S A 1982;79:3265-9.

29. Koyama K, Kagamu H, Miura S, et al. Reciprocal CD4+ T-cell balance of effector CD62Llow CD4+ and CD62LhighCD25+ CD4+ regulatory T cells in small cell lung cancer reflects disease stage. Clin Cancer Res 2008;14:6770-9.

30. Xie D, Marks R, Zhang M, et al. Nomograms Predict Overall Survival for Patients with Small-Cell Lung Cancer Incorporating Pretreatment Peripheral Blood Markers. J Thorac Oncol 2015;10:1213-20.

31. Ribas A. Adaptive Immune Resistance: How Cancer Protects from Immune Attack. Cancer Discov 2015;5:915-9.

32. Ilie M, Long-Mira E, Bence C, et al. Comparative study of the PD-L1 status between surgically resected specimens and matched biopsies of NSCLC patients reveal major discordances: a potential issue for anti-PD-L1 therapeutic strategies. Ann Oncol 2016;27:147-53.
33. Rivalland G, Walkiewicz M, Wright G, et al. Small cell lung cancer: the immune microenvironment and prognostic impact of checkpoint expression. J Clin Oncol 2017;35:8569.

34. Ishii H, Azuma K, Kawahara A, et al. Significance of programmed cell death-ligand 1 expression and its association with survival in patients with small cell lung cancer. J Thorac Oncol 2015;10:426-30.

35. Komiya T, Madan R. PD-L1 expression in small cell lung cancer. Eur J Cancer 2015;51:1853-5.

36. Chang YL, Yang CY, Huang YL, et al. High PD-L1 expression is associated with stage IV disease and poorer overall survival in 186 cases of small cell lung cancers. Oncotarget 2017;8:18021-30.

37. Reck M, Bondarenko I, Luft A, et al. Ipilimumab in combination with paclitaxel and carboplatin as first-line therapy in extensive-disease-small-cell lung cancer: results from a randomized, double-blind, multicenter phase 2 trial. Ann Oncol 2013;24:75-83.

38. Reck M, Luft A, Szczesna A, et al. Phase III Randomized Trial of Ipilimumab Plus Etoposide and Platinum Versus Placebo Plus Etoposide and Platinum in Extensive-Stage Small-Cell Lung Cancer. J Clin Oncol 2016;34:3740-8.

39. Gadgeel SM, Ventimiglia J, Kalemkerian GP, et al. Phase II study of maintenance pembrolizumab (pembro) in extensive stage small cell lung cancer (ES-SCLC) patients (pts). J Clin Oncol 2017;35:8504.

40. Ott PA, Elez E, Hiret S, et al. Pembrolizumab in Patients With Extensive-Stage Small-Cell Lung Cancer: Results From the Phase Ib KEYNOTE-028 Study. J Clin Oncol 2017;35:3823-9.

41. Sequist LV, Chiang A, Gilbert J, et al. Clinical activity, safety and predictive biomarkers results from a phase Ia atezolizumab (atezo) trial in extensive-stage small cell lung cancer. Ann Oncol 2016;27:493-6.

42. Antonia S, Callahan M, Awad M, et al. OA 07.03a - Impact of Tumor Mutation Burden on the Efficacy of Nivolumab or Nivolumab + Ipilimumab in Small Cell Lung Cancer: An Exploratory Analysis of CheckMate 032. 2017 World Conference on Lung Cancer. Abstract OA 07.03a. Presented October 16, 2017.

Cite this article as: Schmid S, Früh M. Immune checkpoint inhibitors and small cell lung cancer: what's new? J Thorac Dis 2018;10(Suppl 13):S1503-S1508. doi: 10.21037/jtd.2018.01.113 